\title{
Regarding "The Interpeduncular Angle: A Practical and Objective Marker for the Detection and Diagnosis of Intracranial Hypotension on Brain MRI"
}

\begin{abstract}
W e carefully read the article by Wang et al. ${ }^{1}$ We are very interested in the method of judging intracranial hypotension by measuring the interpeduncular angle. We hope to apply this method to our clinical work, but the following questions seem to bother us: 1) Specifically, how is intracranial hypotension diagnosed? Is it by judging whether pachymeningeal enhancement or subdural collections or venous engorgement or brain stem slumping in the MR images or a combination of $\geq 2$ of the above? 2) How does the brain stem slumping manifest by imaging only distinguish it from Arnold Chiari malformation? 3) How does one eliminate the influence of head displacement on the interpeduncular angle caused by the displacement of the head during plain MR imaging? If the above problems are solved, the clinical applicability of the interpeduncular angle will be greatly enhanced. We are very grateful to Wang et al for the study, which gave us a new way to evaluate intracranial hypotension on brain MR imaging.
\end{abstract}

http://dx.doi.org/10.3174/ajnr.A6340

\section{REFERENCE}

1. Wang DJ, Pandey SK, Lee DH, et al. The interpeduncular angle: a practical and objective marker for the detection and diagnosis of intracranial hypotension on brain MRI. AJNR Am J Neuroradiol 2019; 40:1299-1303 CrossRef Medline

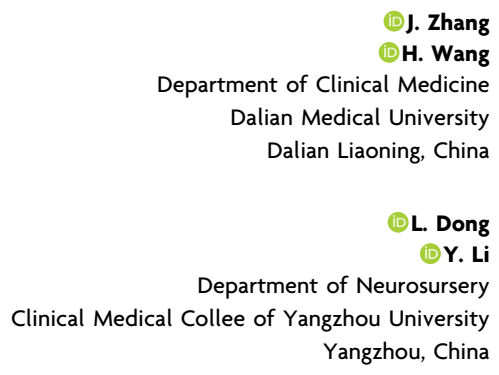

(D). Zhang

partment of Clinical Medicine alian Medical University

L. Dong

Y. Li Deurosursery Yangzhou, China 